\title{
Navigiertes Operieren an der Wirbelsäule
}

\author{
Klaus Wendl, Jochen Franke, Paul Alfred Grützner
}

\section{Zusammenfassung}

Navigiertes Operieren an der Wirbelsäule ist seit 15 Jahren klinische Realität. Neben der CT-gestützten Navigation, die den Vorteil der besten Bildqualität bietet, kommen auch CArm-gestützte Verfahren zur intraoperativen 2- oder 3-dimensionalen Bildakquise zur Anwendung. Insbesondere 3-D-Bildwandler weisen bei guter Bildqualität und hoher Registrierungsgenauigkeit die Option auf, Veränderungen der Anatomie beim frakturierten Wirbel zu adressieren und minimalinvasiv zu instrumentieren. Das bei Weitem häufigste Einsatzgebiet ist die dorsale Stabilisierung mit Pedikelschrauben an der thorakalen und lumbalen Wirbelsäule. Andere Anwendungen wie die Instrumentierung von Halswirbeln, C1-/C2-Fusionen oder ventrale Stabilisierungen sind zahlenmäßig selten. Die klinischen Erfahrungen zeigen einen Trend zur genaueren Platzierung von Pedikelschrauben durch die Anwendung der Navigation, v.a. an HWS und LWS, die Strahlenbelastung für Patient und OP-Personal sinkt. Dafür steigen OPZeiten und die Kosten für die technische Ausstattung. Trotz theoretischer und teilweise belegter praktischer Vorteile der Computernavigation an der Wirbelsäule steht die flächendeckende Anwendung noch aus.

\section{Einleitung}

Bei vielen orthopädisch-unfallchirurgischen Eingriffen ist die direkte visuelle Kontrolle des Operationsgebiets für den Operateur mehr oder weniger stark ein-

OP-JOURNAL 2011; 27: 138-142

(c) Georg Thieme Verlag KG Stuttgart · New York DOI http://dx.doi.org/10.1055/s-0031-1280185

\section{Image-Guided Spine Surgery}

Image-guided spine surgery was introduced to trauma surgery about 15 years ago. Image acquisition can be based on computer tomography, two- or threedimensional C-arm pictures, whereby CT offers the best image quality. Threedimensional image intensifiers show acceptable image quality, have the advantage of an automatic registration process and can consider variations of patient anatomy, e.g., following intraoperative reduction of fractured vertebrae. They also allow minimally invasive operation procedures. Pedicle screw placement in the thoracic and lumbar spines is by far the most common spine surgery in traumatology. Other navigated applications like pedicle screw placement in the cervical spine, $\mathrm{C} 1 / \mathrm{C} 2$ fusions etc. are relatively rare in everyday clinical routine. Clinical studies show a trend towards more precise pedicle screw placement, especially in the lumbar and cervical spines using image-guided spine surgery. The overall radiation exposure can be reduced by means of intraoperative navigation procedures. On the other hand, there is an increase in costs and overall time for surgery. Although imageguided spine surgery has different advantages, both theoretically and clinically proven, it is still no standard method for use in everyday surgery. das gilt bspw. für das Becken, aber auch für die Wirbelkörper der Wirbelsäule. Die häufigste traumatologische Versorgung an der Wirbelsäule ist die Besetzung von Pedikeln thorakolumbaler Wirbelkörper zur dorsalen Instrumentierung bei Frakturen. Die zu instrumentierenden Pedikel sind dabei für das Auge des Operateurs nicht sichtbar und sie haben eine sich in Abhängigkeit von der zu versorgenden Höhe von der Halswirbelsäule bis zur Lendenwirbelsäule kontinuierlich verändernde Form und Lage im Raum. Andererseits ist eine sehr hohe Präzision der Pedikelschraubenplatzierung essenziell. Zum einen liegen in unmittelbarer Nähe der operierten knöchernen Strukturen sensible Weichteile wie Nervenwurzeln, Gefäße und nicht zuletzt das Rückenmark. Fehlplatzierte Schrauben können hier deletäre Folgen mit irreversiblen neurologischen Schäden haben. Darüber hinaus weisen suboptimal platzierte Schrauben eine deutlich niedrigere biomechanische Stabilität auf [14].

Das Einbringen von Pedikelschrauben zur dorsalen Instrumentierung von Wirbelfrakturen stellt v. a. im Bereich der zervikalen und thorakalen Wirbelsäule hohe Anforderungen an das 3-dimensionale Orientierungsvermögen des Operateurs.

Die thorakale Wirbelsäule weist sehr geringe Pedikeldurchmesser auf [16], der Pedikeldurchmesser ist unter Umständen nur wenig größer als der Durchmesser der zu implantierenden Schrauben, entsprechend schwierig ist die korrekte Platzierung. Die 2-dimensionale Visualisierung der zu instrumentierenden Wirbel mit dem klassischen C-Bogen ist häufig problematisch, gerade auch bei degenerativen Veränderungen wie dem Morbus Bechterew, bei sehr adipösen Patienten oder im Bereich der oberen Brustwirbelsäule durch Überlagerung der Schultern.

Zugängen schränkt die Visualisierung weiter ein. Die komplexe 3-dimensionale Struktur von Knochen macht eine Orientierung im Raum zusätzlich schwierig,

geschränkt [6]. Die Ursachen dafür sind vielfältig, so sind viele zentral gelege knorne Strukturen von einem großen komplett abgelöst werden kann oder soll. Auch der Trend zu immer kleineren,

Ziel der Computernavigation ist es, orthopädisch-unfallchirurgische Operatio- 


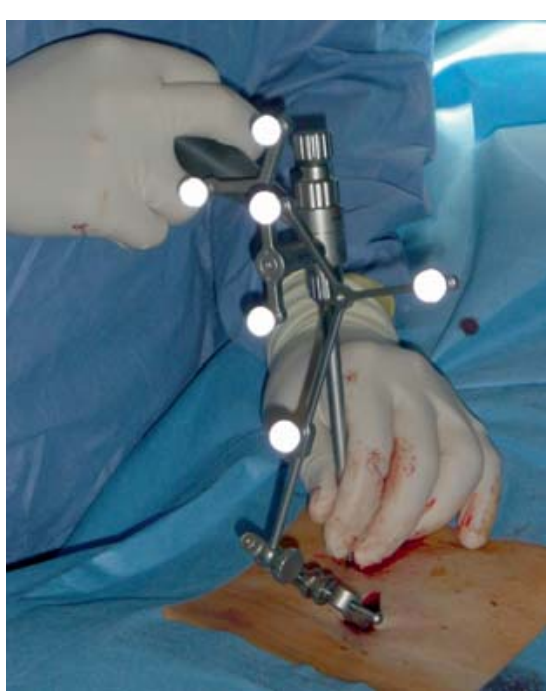

Abb. 1 Navigierte Bohrhülse und dynamische Referenzierungsbasis (DRB) mit lichtreflektierenden Markern zur Navigation, DRB am Wirbelkörper befestigt.

nen möglichst exakt durchzuführen. Das rechnergestützte Operieren bietet darüber hinaus auch die Option, die immer besseren 3-dimensionalen diagnostisch radiologischen Verfahren, bspw. der Computertomografie, in den therapeutischen Prozess zu integrieren.

In den letzten 15 Jahren wurden zahlreiche computernavigierte Operationsverfahren im Bereich der Unfallchirurgie und der orthopädischen Chirurgie mit dem Ziel entwickelt, die Genauigkeit von Eingriffen in komplexen Regionen zu verbessern und die operative Sicherheit für den Patienten zu erhöhen. Aufgrund der oben angeführten Aspekte war es nur folgerichtig, dass als eine der ersten Anwendungen der Computernavigation in der Unfallchirurgie die Pedikelinstrumentierung an der Lendenwirbelsäule bereits 1995 entwickelt wurde [10].

Die Besetzung mit Pedikelschrauben ist weiterhin die häufigste Navigationsform an der Wirbelsäule. Aus diesem Grund soll im Folgenden überwiegend dieses Operationsverfahren bez. Technik und klinischen Ergebnissen näher erläutert werden, auf andere Anwendungen der Navigation an der Wirbelsäule wird orientierend eingegangen.

\section{Technik der Computernavigation an der Wirbelsäule}

\section{Funktionsprinzip}

Bei der intraoperativen Navigation wird typischerweise dem Operateur die Po-

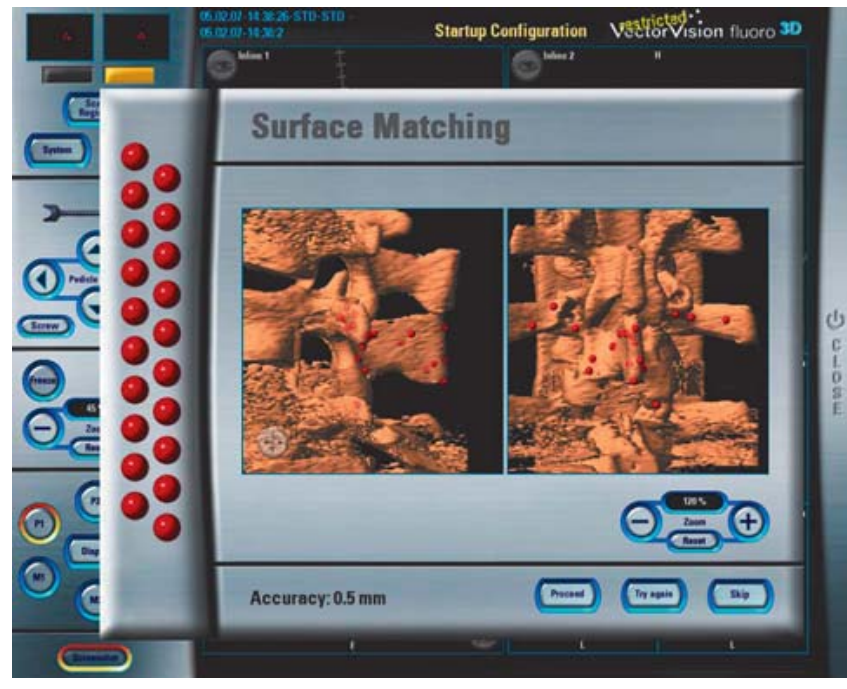

Abb. 2 Surface Matching in einem CT-Datensatz.

sition eines Instruments im Raum in Bezug zur Patientenanatomie, repräsentiert durch prä- oder intraoperative (Röntgen-)Bildgebung, dargestellt (sog. „Tracking“). Meist wird ein Abbild des Instruments auf einem Bildschirm in ein Röntgenbild, seien es klassische 2-dimensionale Durchleuchtungsbilder oder 3-dimensionale Schnittbilder, in Echtzeit überlagert [15].

Die meisten Navigationssysteme verwenden passive optoelektronische Systeme, um die Instrumentenposition kontinuierlich zu erfassen. Eine Kamera sendet dabei Infrarotlicht aus, dieses wird von Kugeln mit einer speziellen reflektierenden Oberfläche, sog. „Markern“, die an allen zu navigierenden Instrumenten befestigt sind, reflektiert und wieder von der Kamera erfasst. Gleichzeitig wird die Position z.B. eines Wirbelkörpers durch eine am Processus spinosus fixierte und ebenfalls mit reflektierenden Markern ausgestattete Referenzbasis (DRB, Dynamic Reference Base) registriert (Abb. 1). Ein Computer errechnet dann online die Lage des oder der Instrumente im Raum in Relation zum reellen Patienten bzw. dessen Anatomie und überlagert ein $\mathrm{Ab}$ bild des Instruments auf ein Bild der zu operierenden Region. Das gelingt experimentell-theoretisch mit einer Genauigkeit von unter $1 \mathrm{~mm}$ [14]. Das erforderliche Bildmaterial kann dem Rechner auf verschiedene Arten zugeführt werden. Voraussetzung ist immer, dass dem Computer mitgeteilt wird, wo im Raum sich der Patient in Relation zum jeweiligen Bild befindet. Dazu können am zu operierenden Knochen und am Röntgengerät, während ein 2-dimensionales Durchleuchtungsbild oder ein 3-dimensionaler Scan eines 3-D-Bildverstärkers akquiriert wird, Referenzbasen angebracht werden und so die Lagebeziehung zwischen Patient und Bild definiert wird. Aber auch präoperativ erzeugte CT-Bilder können verwendet werden, wenn dem Computer die Beziehung zwischen CT-Bild und Patientenanatomie „beigebracht" wird (referenzieren). Dazu werden im Rahmen einer präoperativen Planung in den CT-Schnitten charakteristische Punkte oder Oberflächen auf zu instrumentierenden Wirbeln definiert (paired-point bzw. surface-matching) und später am reellen Patienten erneut mit einem Zeigeinstrument aufgesucht, um den Abgleich zwischen Bilddatensatz und reeller Anatomie herzustellen (Abb. 2). Essenziell ist, dass anschließend die am Knochen befestigte DRB nicht verändert werden darf, da ansonsten das Matching und damit alle davon abhängigen Navigationsmanöver nicht mehr korrekt sind, leider eine häufige Fehlerquelle.

\section{Bildakquise}

Alle Bilddatensätze haben spezifische Vor- und Nachteile. Moderne CT-Bilder haben den Vorteil, dass sie die beste Bildqualität liefern. Die CT-basierte Navigation ist jedoch ein statisches System; intraoperative anatomische Veränderungen des Situs, also wenn sich die Anatomie zwischen Bilderfassung und Operation verändert, wie es gerade bei der Instrumentierung von Wirbelfrakturen durch Repositionsmanöver perioperativ vorkommt, können in die Navigation nicht mit einfließen, was die Anwendung in vielen Fällen limitiert.

Ein weiterer Nachteil der CT-basierten Navigation ist der relativ hohe präoperative Aufwand, insbesondere für die 


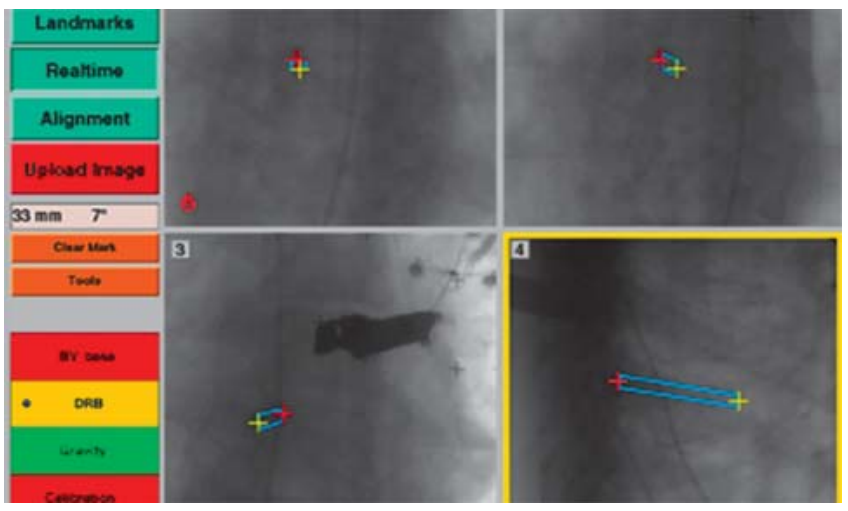

Abb. 3 Navigation in einem 2-D-Datensatz.
Planung der Landmarken für die Referenzierung. Arand et al. [1] weisen auf zahlreiche Fallstricke und Komplikationsmöglichkeiten der CT-Navigation hin. Der verwendete CT-Datensatz muss ein für das jeweilige Navigationssystem kompatibles Datenprotokoll haben [9] und die CT-Daten müssen einlesbar sein, ansonsten ist eine erneute CT-Untersuchung nötig, verbunden mit einer höheren Strahlenbelastung für den Patienten. Der Datentransfer muss vollständig sein, ohne dass einzelne CT-Schichten verloren gehen, sonst ist die Referenzierung, also die Zuordnung von Bilddatensatz zur Patientenrealität, ungenau. Es muss in der Planung auch sichergestellt sein, dass die zum Matching erforderlichen Landmarken am Patienten gut und reproduzierbar zu finden sind; gerade im Bereich der Brustwirbelsäule kann dies schwierig sein [2]. Die Anatomie kann frakturbedingt verändert sein, Landmarken und typische Orientierungspunkte sind unter Umständen frakturbedingt zerstört. Manchmal kann es problematisch sein, den zu matchenden Wirbel korrekt zu erfassen. Man darf sich nicht blind auf ein scheinbar erfolgreiches Matching verlassen!

Ein weiteres Problem ist gerade im Bereich der Notfallversorgung von Frakturen eine begrenzte Planungszeit, auch sind nicht immer die in die Navigation eingearbeiteten Teams vor Ort. Eine CTNavigation erscheint somit angesichts zahlreicher Fallstricke nicht als Anfängereingriff im Dienst.

Den Nachteil von CT-Datensätzen, Veränderungen der Anatomie zwischen Bildakquise und Operation nicht zu erfassen, hat die Navigation mittels konventioneller C-Bogen-Durchleuchtungsbilder (Navigation in 2-D-Datensätzen) nicht, hier können durch intraoperative Aufnahmen jederzeit die aktuellen Gegebenheiten der Patientenanatomie dar- gestellt werden (Abb. 3). Die Beschränkung der Bildinformation auf klassische Durchleuchtungsbilder begrenzt jedoch die Anwendung an komplexen anatomischen Strukturen wie der Wirbelsäule $[5,10]$. Die im Vergleich zum CT deutlich schlechtere Bildqualität ist gerade im Bereich der (oberen) Brustwirbelsäule und der HWS ein limitierender Faktor.

Eine Kombination aus 3-dimensionaler Bildgebung wie im CT mit der Flexibilität der intraoperativen Datenerfassung eines C-Bogens ermöglichen moderne C-Bogen mit der Option einer 3-dimensionalen Bildakquise. Mit modernen 3-D-Bildverstärkern ist es intraoperativ möglich, CT-ähnliche 3-D-Datensätze zu erheben.

Diese 3-dimensionalen Datensätze können direkt an ein Navigationssystem übertragen werden, da während der Datenerfassung die Lage des Bildverstärkers im Raum von der Infrarotkamera des Navigationssystems automatisch erfasst wird (Abb. 4). Das Navigationssystem kann so die Lage und Orientierung des 3-D-Datensatzes im Raum automatisch ohne fehlerbehaftetes und zeitaufwendiges Matching der Patientenanatomie zuordnen und die Position von chirurgischen Instrumenten virtuell im 3-D-Bild anzeigen (Abb.5). Ein weiterer Vorteil der intraoperativen fluoroskopischen Bilddatenerhebung, egal ob 2-D oder 3-D, ist, dass diese Methoden eine minimalinvasive Versorgung ermöglichen, da die Wirbelkörper nicht zum Matching freigelegt werden müssen.

Doch auch diese Methode ist nicht frei von möglichen Fallstricken. Das Volumen des erfassten Gebiets entspricht einem Kubus mit einer Kantenlänge von $12 \mathrm{~cm}$. Für langstreckige Instrumentierungen z.B. an der Brustwirbelsäule sind ggf. mehrere Scans erforderlich. Neuere Entwicklungen wie die von Flatpanels werden hier aber in naher Zukunft Ver-

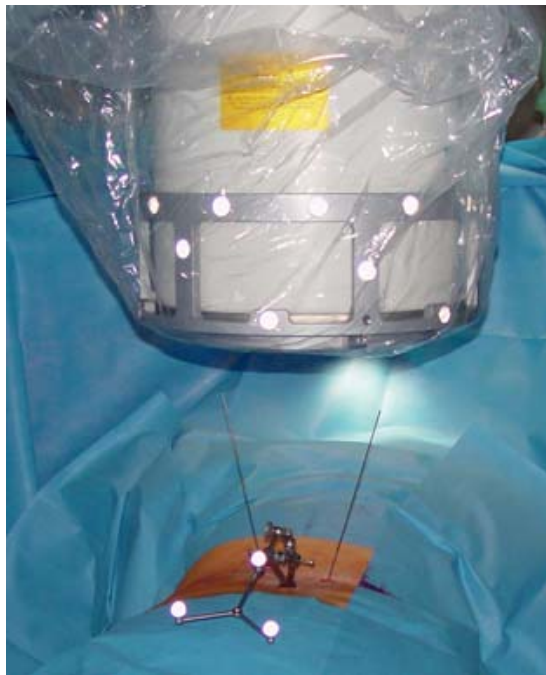

Abb. 4 3-D-Bildverstärker mit reflektierenden Markern zur automatischen Registrierung (oben) und am Wirbelkörper befestigte Referenzierungsbasis (DRB), ebenfalls mit reflektierenden Markern.

besserungen von Bildausschnitt und Bildqualität bringen. Die Bildqualität der Scans ist zwar in den meisten Fällen ausreichend für eine Navigation, erreicht aber nicht die Abbildungsqualität moderner Computertomografen. Die Bildqualität leidet zusätzlich bei sehr adipösen Patienten, Artefakte können durch Veratmung während der Bildakquise (Scan in Atemstillstand ist essenziell!) und eingebrachte Metallimplantate entstehen. Eine Überlegenheit der 3-D-fluoroskopischen Bildakquise vs. Matching von CT-Datensätzen ist aber bisher nicht belegt.

Komplikationsmöglichkeiten der Wirbelsäulennavigation unabhängig vom verwendeten Bilddatensatz sind weiterhin denkbar durch Verschiebungen der an den Wirbelkörpern angebrachten dynamischen Referenzierungsbasen, gerade bei Wirbeln mit frakturierten Dornfortsätzen kann das Anbringen der DRB schwierig sein. Auch bei der Navigation mehrerer Wirbelkörper kann es zu Ungenauigkeiten kommen, je weiter der zu instrumentierende Wirbel vom DRB tragenden Wirbelkörper entfernt ist.

Die 3 häufigsten Fehlerursachen bei der Navigation sind die nicht korrekte Registrierung der Patientenanatomie, Probleme bei der Befestigung des Referenzierungskörpers am Patienten und das Verbiegen von Instrumenten während des Eingriffs [4]. 

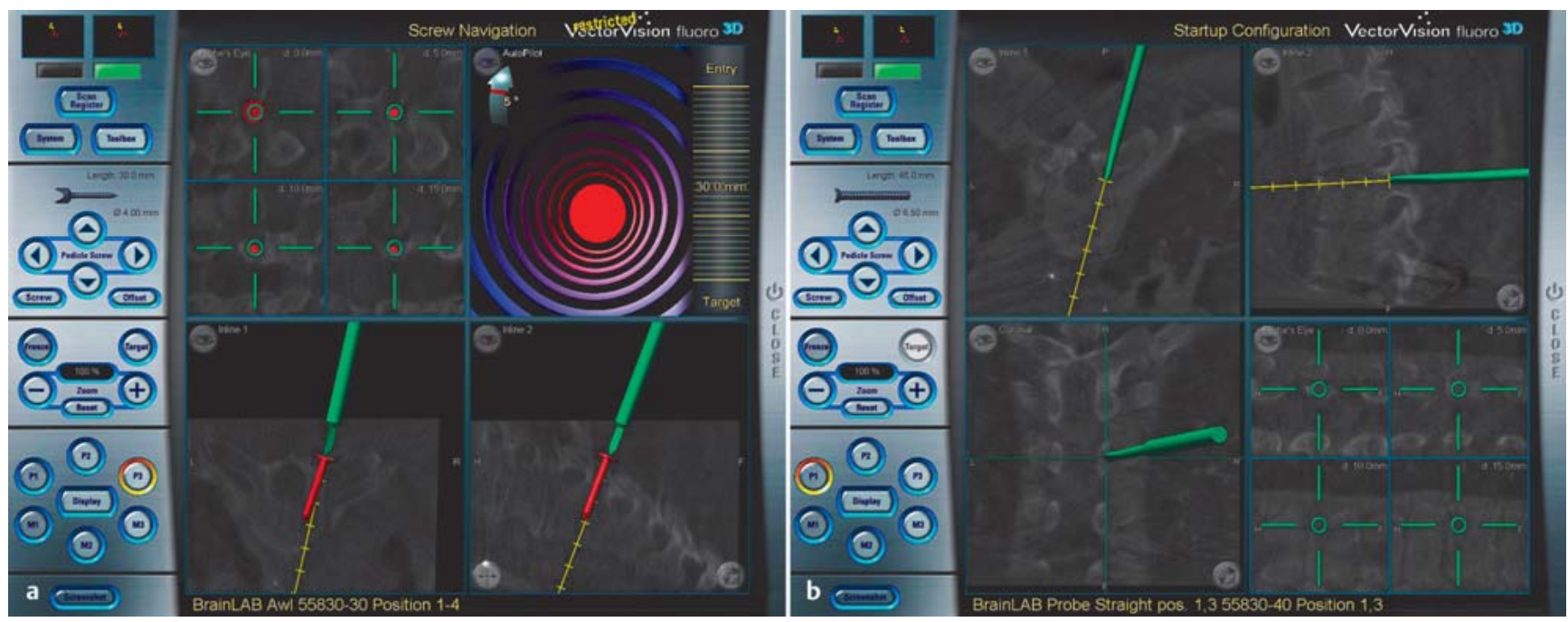

Abb. $\mathbf{5} \mathbf{a}$ und $\mathbf{b}$ Navigation in 3-D-Bildverstärker-Datensatz.

Da alle Navigationsverfahren trotz „hightech" nicht frei von Fehlerquellen sind, muss vor der Gefahr einer falschen virtuellen Sicherheit gewarnt werden, v.a. nach einigen erfolgreichen Anwendungen der Navigation [1].

\section{Klinische Erfahrungen in der Anwen- dung der Navigation an der Wirbelsäule}

\section{Pedikelinstrumentierung an der \\ Brust- und Lendenwirbelsäule}

Die Navigation an der Brust- und Lendenwirbelsäule ist primär mit der Zielsetzung entwickelt worden, die Genauigkeit der Besetzung von Pedikeln mit Schrauben zu verbessern. Hintergrund dieser Bestrebungen waren nicht zuletzt Hinweise aus der Literatur, die in einem hohen Prozentsatz Fehllagen von Pedikelschrauben zeigten. Fehllagen in bis zu 30\% an der Lendenwirbelsäule und bis zu 55\% an der Brustwirbelsäule sind beschrieben $[3,12]$. In zahlreichen experimentellen und klinischen Untersuchungen wurde die Präzision der Pedikelinstrumentierung mithilfe der Computernavigation untersucht.

Dass die Navigation von Pedikelschrauben technisch gut reproduzierbar durchführbar ist, zeigen viele Studien. Insgesamt findet sich auch eine relativ niedrige Rate an Fehlplatzierungen, je nach Untersuchung zwischen $0 \%$ und $8 \%[5,14]$.

Vergleichende Untersuchungen von navigiert und nicht navigiert eingebrachten Schrauben ergeben jedoch ein uneinheitliches Bild, teilweise sind für die Navigation bessere Ergebnisse, teilweise aber auch nur gleich gute Ergebnisse beschrieben. Metaanalysen zeigen für die Lendenwirbelsäule und den thorakolumbalen Übergang einen leichten Trend zu besseren Ergebnissen mithilfe der Navigation, für die Brustwirbelsäule jedoch nicht. Letzteres verwundert umso mehr, da gerade die kleinen Pedikeldurchmesser zumindest theoretisch Vorteile durch die Navigation zeigen sollten. Ein Dilemma mag dabei darin bestehen, dass die intensive Auseinandersetzung mit der Problematik fehlplatzierter Pedikelschrauben durch die Einführung der Navigation zeigte, dass eine einheitliche Definition, ab wann eine Schraube als „fehlplatziert“ gilt, so nicht existiert [8], was die Interpretation bzw. den Vergleich von verschiedenen Untersuchungen wesentlich erschwert. Ebenso schwer ist es zu definieren, welche klinische Bedeutung welches Ausmaß z.B. einer medialen oder lateralen Perforation eines Pedikels hat, da dies klinisch in den meisten Fällen unerkannt und folgenlos bleibt. Was ist tolerabel und was nicht? Viele Studien beziehen zudem ihre Daten aus Patientengruppen mit degenerativen Erkrankungen [11], die entsprechenden Daten sind auch aus den oben angeführten Gründen nicht immer auf die Situation in der Traumaversorgung zu übertragen.

Fast alle Untersuchungen zeigen, dass auch die Computernavigation keine Garantie für die Vermeidung von Fehlplatzierungen darstellt!

Ebenso uneinheitlich ist die Datenlage bez. der Frage, welche Bildmodalität (2-D-C-Bogen, 3-D-Bildwandler, CT-Datensätze) in der klinischen Praxis zu ge- naueren Ergebnissen führt. Hier gibt es zumindest an der BWS einen Trend zur Verwendung von CT- oder 3-D-Bildwandlerbildern aufgrund der schlechteren Darstellbarkeit zumindest der oberen BWS im 2-D-Bild. In vielen Publikationen wird eine nicht zu vernachlässigende Anzahl von Abbrüchen der Navigation aus technischen Gründen angegeben, sodass auch bei geplanter Navigation immer die konventionelle Technik beherrscht werden muss.

\section{Röntgenstrahlenexposition}

Die Instrumentierung von Pedikeln ist typischerweise mit einer relativ hohen Exposition gegenüber Röntgenstrahlung sowohl für den Patienten als auch für das OP-Personal, die instrumentierende OP-Pflege und die Chirurgen verbunden.

Hier konnte in mehreren Untersuchungen [5] gezeigt werden, dass die Navigation zu einer deutlichen Reduktion der Strahlenbelastung führt. Die größten Vorteile scheint hier, insbesondere für die beteiligten Teams, die intraoperative 3-D-Durchleuchtung zu bieten [14].

\section{Zeitbedarf}

Navigation erfordert Zeit, sowohl prä- als auch intraoperativ. Gerade während der initialen Lernkurve ist mit einer Verlängerung der OP-Zeit zu rechnen [11], gerade auch in der Notfallsituation, wenn keine in der Navigation erfahrenen Teams verfügbar sind. Der z.T. erhebliche Zeitaufwand der präoperativen Planung und des intraoperativen Matching bei Verwendung von CT-Datensätzen ist nicht zu vernachlässigen. 


\section{Kosten/Effektivität}

Eine Untersuchung aus den USA hat errechnet, dass die Navigation bei hohen Operationszahlen von Pedikelinstrumentierungen durch die potenzielle Verminderung von Komplikationen durchaus zu einer Reduktion der Gesamtkosten führen kann [13]. Inwieweit sich diese Ergebnisse auf den deutschen Klinikalltag übertragen lassen, sei dahingestellt. Fakt ist jedenfalls, dass die Implementierung eines Navigationssystems mit einem 3-D-Bildwandler zu Kosten von ca. 400000 EUR führt; ob sich diese amortisieren oder sich im DRG-System abbilden lassen, bleibt zweifelhaft.

\section{Weitere Anwendungen der Navigation an der Wirbelsäule}

Auch die Halswirbelsäule ist bez. einer Pedikelinstrumentierung, mehr noch als die BWS, als High-Risk-Area zu klassifizieren, die anatomischen Strukturen sind klein, die wirbelkörpernah verlaufende A. vertebralis ein zusätzlicher Risikofaktor.

Der Korridor zur sicheren Platzierung von Schrauben ist eng. Neben Pedikelinstrumentierungen sind bspw. auch transartikuläre Verschraubungen $\mathrm{C} 1 / \mathrm{C} 2$ nach Magerl und transpedikuläre C2Schraubenosteosynthesen navigiert beschrieben. In Studien konnte die technische Machbarkeit gut belegt werden, sichere Hinweise auf einen Vorteil der Navigation gibt es jedoch bisher nicht [12], auch wenn der Trend deutlich eine sicherere Versorgung mit reduzierter Rate an Fehllagen von Pedikelschrauben [7] gerade bei engen anatomischen Verhältnissen nahelegt.

Das Matching von CT-Bildern an der HWS ist technisch anspruchsvoll, ebenso die sichere Fixierung der DRB am Wirbel. Zudem ist die Beweglichkeit der HWS deutlich höher als thorakolumbal, eine DRB am Nachbarwirbel oft zu ungenau. Überwiegend wird in CT-Bildern navigiert. Die Bildqualität von intraoperativen 3-D-Scans ist nicht immer ausreichend. Der große Vorteil der Navigation kann hier sein, präoperativ zu planen, ob und welche Zielkorridore die individuelle Patientenanatomie überhaupt ermöglicht. Zusammenfassend ist die Navigation an der HWS, noch mehr als thorakolumbal, nur an wenigen spezialisierten Zentren, typischerweise im Rahmen von Studien, eingeführt, von einer Routineanwendung ist sie, trotz theoretischer Vorteile, weit entfernt.
Auch die Platzierung von Cages und Knochenblöcken zur ventralen Versorgung von Wirbelkörpern [9], die Kyphoplastie und Vertebroplastie, die operative Entfernung von Tumoren und ein Bandscheibenersatz können navigiert durchgeführt werden. Diese Methoden sind teilweise technisch anspruchsvoll und haben überwiegend einen eher experimentellen Charakter, Belege für eine Verbesserung der Ergebnisse gegenüber nicht navigiertem Vorgehen stehen aus.

\section{Schlussfolgerung}

Dass im Bereich der Wirbelsäule die technische Durchführung der Navigation möglich ist, konnte in zahlreichen Untersuchungen, v.a. für die Pedikelinstrumentierung thorakolumbal, belegt werden. Weniger klar zu beantworten ist die Frage, wo es sinnvoll ist zu navigieren. Hier gibt es theoretische, aber auch klinische Hinweise, dass zervikal und lumbal Vorteile vorhanden sind, thorakal steht der Nachweis noch aus. Die uneinheitliche Studienlage mag ein Grund dafür sein, dass die Navigation an der Wirbelsäule, wie fast alle neuen Techniken, nach großer anfänglicher Begeisterung und nachdem die Durchführbarkeit belegt war, mittlerweile eine realistischere und relativierte Bedeutung erfahren hat. Momentan ist die Navigation an der Wirbelsäule als zusätzliche Hilfe für den erfahrenen Operateur zu sehen, überwiegend bei schwierigen anatomischen Verhältnissen wie an der HWS oder stark degenerativ oder osteoporotisch veränderten traumatisierten Wirbelsäulen, bei denen eine konventionelle intraoperative 2-D-Darstellung mit dem C-Bogen schwierig ist. Die geringere Röntgenbelastung durch die Navigation für Patient und Personal kann als belegt gelten. Die Navigation bietet zudem eine gute Möglichkeit zur Planung und Simulation von Operationen, auch im Rahmen der Ausbildung. Es gibt aber zurzeit keine flächendeckende Navigation an der Wirbelsäule in Deutschland und man kann sicher nicht von einem Standard sprechen! Abzuwarten bleibt, inwieweit eine verbesserte intraoperative Bildgebung wie 3-D-C-Bögen mit Flatpanels, einfachere und automatisierte Matching-Algorithmen, automatisierte Fusion von intraoperativen und präoperativen Bildern oder auch eine Kombination der Navigation mit der Robotik zu einer Ausweitung computerunterstützter OP-Verfahren an der Wirbelsäule führt.

\section{Literatur}

${ }^{1}$ Arand M, Kinzl L, Gebhard F. Komplikationen navigierter Eingriffe an der Wirbelsäule. Trauma Berufskrankheit 2005; 7 (Suppl.2): S311-S316

2 Geerling J, Gosling T, Gosling A et al. Navigated pedicle screw placement: experimental comparison between CT- and 3D fluoroscopybased techniques. Comput Aided Surg 2008; 13: 157-166

${ }^{3}$ Gertzbein SD, Robbins SE. Accuracy of pedicular screw placement in vivo. Spine 1990; 15: 11-14

${ }^{4}$ Grützner PA, Wentzensen A. Navigationssystem und Bildgebung, Setup, Wartung und Bedienung. OP-Journal 2005; 21: 46-50

${ }^{5}$ Grützner PA, Beutler T, Wendl Ket al. Navigation an der Brust- und Lendenwirbelsäule mit dem 3D Bildwandler. Chirurg 2004; 75: $967-$ 975

${ }^{6}$ Grützner PA. Navigierte Operationstechniken. Trauma Berufskrankh 2010; 12: 70-77

7 Ito H, Neo M, Yoshida M et al. Efficacy of computer-assisted pedicle screw insertion for cervical instability in RA patients. Rheumatol Int 2007; 27: 567-574

${ }^{8}$ Kosmopoulos V, Schizas C. Pedicle screw placement accuracy: a meta-analysis. Spine 2007; 32: E111-E120

${ }^{9}$ Kraus M, Schultheiß M, Gebhard F. Wirbelsäulenverletzungen und Unfallfolgezustände. Trauma Berufskrankh 2010; 12: 78-87

10 Nolte LP, Visarius H, Arm E et al. Computeraided fixation of spinal implants. J Image Guided Surg 1995; 1: 88-93

11 Schleicher P, Scholz M, Schnake K et al. Navigation bei thorakolumbalen Wirbelsäulenverletzungen. Trauma Berufskrank 2009; 11: 100-105

12 Tjardes T, Shafizadeh S, Rixen D et al. Imageguided spine surgery: state of the art and future directions. Eur Spine J 2010; 19: 25-45

${ }^{13}$ Watkins 4th RG, Gupta A, Watkins 3rd RG. Cost-effectiveness of image-guided spine surgery. Open Orthop J 2010; 4: 228-233

14 Wendl K, von Recum J, Wentzensen A et al. IsoC3D-gestützte navigierte Implantation von Pedikelschrauben an BWS und LWS. Unfallchirurg 2003; 106: 907-913

${ }^{15}$ Wendl $K$, von Recum J, Wentzensen A et al. Computerassistiertes Operieren in der Unfallchirurgie. Chirurgische Praxis 2005; 65: 57-67

${ }^{16}$ Zindrick MR, Wiltse LL, Doornik A et al. Analysis of the morphometric characteristics of the thoracic and lumbar pedicles. Spine 1987; 12: $160-166$

\section{Dr. med. Klaus Wendl \\ Oberarzt \\ Dr. med. Jochen Franke \\ Assistenzarzt}

Prof. Dr. med. Paul Alfred Grützner

Ärztlicher Direktor

BG-Unfallklinik Ludwigshafen

Klinik für Unfall- und

Wiederherstellungschirurgie

an der Universität Heidelberg

Ludwig-Guttmann-Straße 13

67071 Ludwigshafen

kwendl@bgu-ludwigshafen.de 